\title{
Calderón's reproducing Formula For q-Bessel operator
}

\author{
Belgacem Selmi \\ Faculté des Sciences de Bizerte, \\ Département de Mathématiques, \\ 7021 Zarzouna, Tunisie. \\ belgacem.selmi@fsb.rnu.tn
}

\begin{abstract}
In this paper a Calderón-type reproducing formula for q-Bessel convolution is established using the theory of q-Bessel Fourier transform [13, 17, obtained in Quantum calculus.
\end{abstract}

\section{RESUMEN}

En este trabajo se prueba una fórmula de tipo Calderón para convolución q-Bessel, usando la teoría de q-Bessel transformada de Fourier [13, 17], obtenida en cálculo cuántico.

Keywords and Phrases: q-Calderon, q-Calculus, q-Bessel Convolution, q-Fourier Bessel transform, q-Measure.

2010 AMS Mathematics Subject Classification: 05A30, 33DXX, 44A15, 33D15. 


\section{Introduction}

Calderón's formula [1] involving convolutions related to the Fourier transform is useful in obtaining reconstruction formula for wavelet transform, in decomposition of certain spaces and in characterization of Besov spaces [6, 8, 10]. Calderón's reproducing formula was also established for Bessel operator [4, 5]. This work is a continuation of a last work [9], and we establish formula for q-Bessel convolution for both functions and measures witch generalize the above one.

In the classical case this formula is expressed for a suitable function $f$ as follows:

$$
f(x)=\int_{0}^{\infty}\left(g_{t} * h_{t} * f\right)(x) \frac{d t}{t}
$$

where $g, h \in L^{2}(\mathbb{R})$ and $g_{t}(x)=\frac{1}{t} g\left(\frac{x}{t}\right), h_{t}(x)=\frac{1}{t} h\left(\frac{x}{t}\right), t>0$ satisfying

$$
\int_{0}^{\infty} \hat{g}(x t) \hat{h}(x t) \frac{d x}{x}=1, \text { for all } t \in \mathbb{R} \backslash\{0\},
$$

where $\hat{g}$ and $\hat{h}$ is the usual Fourier transform of $g$ and $h$ on $\mathbb{R}$.

If $\mu$ is a finite Borel measure on the real line $\mathbb{R}$, identity (1) has natural generalization as follow

$$
f(x)=\int_{0}^{\infty}\left(f * \mu_{t}\right)(x) \frac{d t}{t}
$$

where $\mu_{t}$ is the dilated measure of $\mu$ under some restriction on $\mu$, the $L^{p}$-norm of (2) has proved in 2]. A general form of (2) has been investigated in [3].

In this paper we study similar questions when in (11) and (2) the classical convolution $*$ is replaced by the $\mathrm{q}$-Bessel convolution $*_{\alpha, \mathrm{q}}$ on the half line generated by the q-Bessel operator defined by

$$
\Delta_{q, \alpha} f(x)=\frac{1}{x^{2 \alpha+1}} D_{q}\left[x^{2 \alpha+1} D_{q} f\right]\left(q^{-1} x\right) .
$$

In this paper we prove that, for $\varphi$ and $\psi \in \mathrm{L}_{\alpha, \mathbf{q}}^{1}\left(\mathbb{R}_{\mathbf{q},+}, \mathrm{d}_{\mathbf{q}} \sigma(x)\right)$ satisfying

$$
\int_{0}^{\infty} \mathcal{F}_{\alpha, q}(\varphi)(\xi) \mathcal{F}_{\alpha, q}(\psi)(\xi) \frac{d_{q} \xi}{\xi}=1
$$

we have

$$
f(x)=\int_{0}^{\infty}\left(f *_{\alpha, q} \varphi_{t} *_{\alpha, q} \psi_{t}\right)(x) \frac{d_{q} t}{t}, \quad f \in L_{\alpha, q}^{1}\left(\mathbb{R}_{q,+}, d_{q} \sigma(x)\right) .
$$

where $d_{q} \sigma(x)=\frac{(1+q)^{-\alpha}}{\Gamma_{q^{2}}(\alpha+1)} x^{2 \alpha+1} d_{q} x=b_{\alpha, q} x^{2 \alpha+1} d_{q} x, \quad \varphi_{t}(x)=\frac{1}{t^{2 \alpha+2}} \varphi\left(\frac{x}{t}\right)$.

In particular for $\varphi \in \mathrm{L}_{\alpha, \mathrm{q}}^{1}\left(\mathbb{R}_{\mathrm{q},+}, \mathrm{d}_{\mathrm{q}} \sigma(x)\right)$ such that

$$
\int_{0}^{\infty}\left[\mathcal{F}_{\alpha, q}(\varphi)(\xi)\right]^{2} \frac{d_{q} \xi}{\xi}=1,
$$


and for a suitable function $f$, put

$$
f^{\varepsilon, \delta}(x)=\int_{\varepsilon}^{\delta}\left(f *_{\alpha, q} \varphi_{t} *_{\alpha, q} \varphi_{t}\right)(x) \frac{d_{q} t}{t}
$$

then

$$
\left\|f^{\varepsilon, \delta}-f\right\|_{2, \alpha, q} \longrightarrow 0 \quad \text { as } \varepsilon \rightarrow 0 \text { and } \delta \rightarrow \infty .
$$

In the case $f \in \mathrm{L}_{\alpha, \mathbf{q}}^{1}\left(\mathbb{R}_{\mathbf{q},+}, \mathrm{d}_{\mathbf{q}} \sigma(x)\right)$ such that $\mathcal{F}_{\alpha, \mathbf{q}} f \in \mathrm{L}_{\alpha, \mathbf{q}}^{1}\left(\mathbb{R}_{\mathbf{q},+}, \mathrm{d}_{\mathbf{q}} \sigma(x)\right)$ one has

$$
\lim _{\substack{\varepsilon \rightarrow 0 \\ \delta \rightarrow \infty}} f^{\varepsilon, \delta}(x)=f(x), x \in \mathbb{R} .
$$

Then we prove that for $\mu \in \mathcal{M}^{\prime}\left(\mathbb{R}_{\mathbf{q},+}\right)$, such that the q-integral

$$
c_{\mu, \alpha, q}=\int_{0}^{\infty} \mathcal{F}_{\alpha, q}(\mu)(\lambda) \frac{d_{q} \lambda}{\lambda}
$$

is finite. Then for all $f \in \mathrm{L}_{\alpha, \mathbf{q}}^{2}\left(\mathbb{R}_{\mathbf{q},+}, \mathrm{d}_{\mathbf{q}} \sigma(x)\right)$, we have

$$
\begin{aligned}
& \lim _{\varepsilon \rightarrow 0} f^{\varepsilon, \delta}=c_{\mu, \alpha, q} f . \\
& \delta \rightarrow \infty
\end{aligned}
$$

where the limit is in $\mathrm{L}_{\alpha, \mathbf{q}}^{2}\left(\mathbb{R}_{\mathbf{q},+}, \mathrm{d}_{\mathbf{q}} \sigma(x)\right)$. And if $\mu \in \mathcal{M}^{\prime}\left(\mathbb{R}_{\mathbf{q},+}\right)$ is such that the q-integral

$$
\int_{0}^{\infty}|\mu([0, y])| \frac{d_{q} y}{y}
$$

is finite, for all $f \in \mathrm{L}_{\alpha, \mathbf{q}}^{2}\left(\mathbb{R}_{\mathbf{q},+}, \mathrm{d}_{\mathrm{q}} \sigma(x)\right)$

$$
\lim _{\substack{\varepsilon \rightarrow 0 \\ \delta \rightarrow \infty}} f^{\varepsilon, \delta}=c_{\mu, \alpha, q} f, \quad \text { in } L_{\alpha, q}^{2}\left(\mathbb{R}_{q,+}, d_{q} \sigma(x)\right) .
$$

The outline of this paper is as follows: In Section 2, basic properties of q-Bessel transform on $\mathbb{R}_{\mathbf{q}}$ of functions and bounded measure and its underlying $\mathbf{q}$-convolution structure are called and introduced here. In Section 3, we give the first main result of the paper, the q-Calderon's reproducing formula for functions. Section 4 is consecrate to establish the same result as in section 3 for finite measures.

\section{Preliminaries}

In this section we recall some basic result in harmonic analysis related to the q-Bessel Fourier transform. Standard reference here is Gasper \& Rahman [7]. 
For $a, q \in \mathbb{C}$ the $q$-shifted factorial $(a ; q)_{k}$ is defined as a product of $k$ factors:

$$
(a ; q)_{k}=(1-a)(1-a q) \ldots\left(1-a q^{k-1}\right), \quad k \in \mathbb{N}^{*} ; \quad(a ; q)_{0}=1 .
$$

If $|q|<1$ this definition remains meaningful for $k=+\infty$ as a convergent infinite product:

$$
(a ; q)_{\infty}=\prod_{k=0}^{\infty}\left(1-a q^{k}\right) .
$$

We also write $\left(a_{1}, \ldots, a_{r} ; q\right)_{k}$ for the product of $r q$-shifted factorials:

$$
\left(a_{1}, \cdots, a_{r} ; q\right)_{k}=\left(a_{1} ; q\right)_{k} \ldots\left(a_{r} ; q\right)_{k} \quad(k \in \mathbb{N} \text { or } k=\infty)
$$

A q-hypergeometric series is a power series (for the moment still formal) in one complex variable $z$ with power series coefficients which depend, apart from $q$, on $r$ complex upper parameters $a_{1}, \ldots, a_{r}$ and $s$ complex lower parameters $b_{1}, \ldots, b_{s}$ as follows:

$r \varphi_{s}\left(a_{1}, \cdots, a_{r} ; b_{1}, \cdots, b_{s} ; q, x\right)=\sum_{k=0}^{\infty} \frac{\left(a_{1}, \cdots, a_{r} ; q\right)_{k}}{\left(b_{1}, \cdots, b_{s} ; q\right)_{k}(q ; q)_{k}}\left[(-1)^{k} q^{\frac{k(k-1)}{2}}\right]^{1+s-r} x^{k} \quad($ for $r, s \in \mathbb{N})$

\section{$2.1 \quad$ q-Exponential series}

$$
\begin{aligned}
& e_{\mathrm{q}}(z)={ }_{1} \varphi_{0}(0 ;-; \mathrm{q}, z)=\sum_{k=0}^{\infty} \frac{z^{\mathrm{k}}}{(\mathrm{q} ; \mathrm{q})_{\mathrm{k}}}=\frac{1}{(z ; \mathrm{q})_{\infty}} \quad(|z|<1) \\
& \mathrm{E}_{\mathrm{q}}(z)={ }_{0} \varphi_{0}(-;-; \mathrm{q},-z)=\sum_{\mathrm{k}=0}^{\infty} \frac{\mathrm{q}^{\frac{1}{2} \mathrm{k}(\mathrm{k}-1)} z^{\mathrm{k}}}{(\mathrm{q} ; \mathrm{q})_{k}}=(-z ; \mathrm{q})_{\infty} \quad(z \in \mathbb{C}) .
\end{aligned}
$$

\section{2 q-Derivative and q-Integral}

The q-derivative of a function $f$ given on a subset of $\mathbb{R}$ or $\mathbb{C}$ is defined by:

$$
D_{q} f(x)=\frac{f(x)-f(q x)}{(1-q) x} \quad(x \neq 0, q \neq 0)
$$

where $x$ and $q x$ should be in the domain of $f$. By continuity we set $\left(D_{q} f\right)(0)=f^{\prime}(0)$ provided $f^{\prime}(0)$ exists.

The q-shift operators are

$$
\left(\Lambda_{q} f\right)(x)=f(q x), \quad\left(\Lambda_{q}^{-1} f\right)(x)=f\left(q^{-1} x\right) .
$$


For $a \in \mathbb{R} \backslash\{0\}$ and a function $f$ given on $(0, a]$ or $[a, 0)$, we define the $q$-integral by

$$
\int_{0}^{a} f(x) d_{q} x=(1-q) a \sum_{n=0}^{\infty} f\left(a q^{n}\right) q^{n}
$$

provided the infinite sum converges absolutely (for instance if $f$ is bounded). If $F(a)$ is given by the left-hand side of (21) then $D_{q} F=f$. The right-hand side of (21) is an infinite Riemann sum.

For a q-integral over $(0, \infty)$ we define

$$
\int_{0}^{\infty} f(x) d_{q} x=(1-q) \sum_{-\infty}^{+\infty} f\left(q^{k}\right) q^{k}
$$

Note that for $n \in \mathbb{Z}$ and $a \in \mathbb{R}_{\mathrm{q}}$, we have

$$
\int_{0}^{\infty} f\left(q^{n} x\right) d_{q} x=\frac{1}{q^{n}} \int_{0}^{\infty} f(x) d_{q} x, \quad \int_{0}^{a} f\left(q^{n} x\right) d_{q} x=\frac{1}{q^{n}} \int_{0}^{a q^{n}} f(x) d_{q} x .
$$

The $q$-integration by parts is given for suitable functions $f$ and $g$ by:

$$
\int_{a}^{b} f(x) D_{q} g(x) d_{q} x=[f(x) g(x)]_{a}^{b}-\int_{a}^{b} D_{q} f(x) g(x) d_{q} x .
$$

The $\mathrm{q}$-Logarithm $\log _{\mathrm{q}}$ is given by [19]

$$
\log _{q} x=\int \frac{d_{q} x}{x}=\frac{1-q}{\log q} \log x .
$$

For all $a, b \in q^{\mathbb{Z}}, a<b$

$$
\log _{q}(b / a)=(1-q) \sum_{k: a \leq q^{k} \leq b} 1 .
$$

The improper integral is defined in the following way

$$
\int_{0}^{\infty / A} f(x) d_{q} x=(1-q) \sum_{-\infty}^{+\infty} f\left(\frac{q^{n}}{A}\right) \frac{q^{n}}{A} .
$$

We remark that for $n \in \mathbb{Z}$, we have

$$
\int_{0}^{\infty / q^{n}} f(x) d_{q} x=\int_{0}^{\infty} f(x) d_{q} x
$$

The following property holds for suitable function $f$

$$
\int_{0}^{\infty} \int_{0}^{x} f(x, y) d_{q} y d_{q} x=\int_{0}^{\infty} \int_{q y}^{\infty} f(x, y) d_{q} x d_{q} y .
$$




\subsection{The q-gamma function}

The q-gamma function is defined by [7, 16 .

$$
\begin{aligned}
\Gamma_{\mathrm{q}}(z) & =\frac{(\mathrm{q} ; \mathrm{q})_{\infty}}{\left(\mathrm{q}^{z} ; \mathrm{q}\right)_{\infty}}(1-\mathrm{q})^{1-z}, \quad 0<\mathrm{q}<1 ; z \neq 0,-1,-2, \ldots \\
& =\int_{0}^{(1-\mathrm{q})^{-1}} \mathrm{t}^{z-1} \mathrm{E}_{\mathrm{q}}(-(1-\mathrm{q}) \mathrm{q} \mathrm{t}) \mathrm{d}_{\mathrm{q}} \mathrm{t}, \quad(\operatorname{Re} z>0)
\end{aligned}
$$

moreover the q-duplication formula holds

$$
\Gamma_{\mathrm{q}}(2 z) \Gamma_{\mathrm{q}^{2}}\left(\frac{1}{2}\right)=(1+\mathrm{q})^{2 z-1} \Gamma_{\mathrm{q}}^{2}(z) \Gamma_{\mathrm{q}^{2}}\left(z+\frac{1}{2}\right) .
$$

\subsection{Some q-functional spaces}

We begin by putting

$$
\mathbb{R}_{\mathbf{q},+}=\left\{+\mathbf{q}^{\mathrm{k}}, \mathrm{k} \in \mathbb{Z}\right\}, \quad \widetilde{\mathbb{R}}_{\mathbf{q},+}=\left\{+\mathbf{q}^{\mathrm{k}}, \mathrm{k} \in \mathbb{Z}\right\} \cup\{0\}
$$

and we denote by

- $\mathrm{L}_{\alpha, \mathbf{q}}^{\mathrm{p}}\left(\mathbb{R}_{\mathbf{q},+}\right), \quad p \in\left[1,+\infty\left[,\left(\operatorname{resp} . \mathrm{L}_{\alpha, \mathbf{q}}^{\infty}\left(\mathbb{R}_{\mathbf{q},+}\right)\right)\right.\right.$ the space of functions $f$ such that,

$$
\begin{aligned}
& \|f\|_{p, \alpha, q}=\left(\int_{0}^{\infty}|f(x)|^{p} d_{q} \sigma(x)\right)^{\frac{1}{p}}<+\infty . \\
& \text { (resp. } \left.\|f\|_{\infty, q}=\text { ess } \sup _{x \in \mathbb{R}_{q,+}}|f(x)|<+\infty\right) .
\end{aligned}
$$

- $\mathcal{S}_{\mathrm{q}, *}\left(\mathbb{R}_{\mathrm{q}}\right)$ the $\mathrm{q}$-analogue of Schwartz space of even functions defined on $\mathbb{R}_{\mathrm{q}}$ such that $\mathrm{D}_{\mathrm{q}, \mathrm{x}}^{\mathrm{k}} \mathrm{f}(\mathrm{x})$ is continuous in 0 for all $k \in \mathbb{N}$ and

$$
\mathrm{N}_{\mathrm{q}, \mathrm{n}, \mathrm{k}}(\mathrm{f})=\sup _{\mathrm{x} \in \mathbb{R}_{\mathrm{q}}}\left|\left(1+x^{2}\right)^{\mathrm{n}} \mathrm{D}_{\mathrm{q}, \mathrm{x}}^{\mathrm{k}} \mathrm{f}(\mathrm{x})\right|<+\infty .
$$

- The q-analogue of the tempered distributions is introduced in [12] as follow:

(i) A q-distribution $T$ in $\mathbb{R}_{q}$ is said to be tempered if there exists $C_{q}>0$ and $k \in \mathbb{N}$ such that:

$$
|\langle T, f\rangle| \leq \mathrm{C}_{\mathrm{q}} \mathrm{N}_{\mathrm{q}, \mathrm{n}, \mathrm{k}}(\mathrm{f}) ; \quad \mathrm{f} \in \mathcal{S}_{\mathrm{q}, *}\left(\mathbb{R}_{\mathrm{q}}\right) .
$$

(ii) A linear form $\mathrm{T}: \mathrm{S}_{\mathrm{q}, *}\left(\mathbb{R}_{\mathrm{q}}\right) \longrightarrow \mathbb{C}$ is said continuous if there exist $\mathrm{C}_{\mathrm{q}}>0$ and $\mathrm{k} \in \mathbb{N}$ such that:

$$
|\langle T, f\rangle| \leq C_{q} N_{q, n, k}(f) ; \quad f \in \mathcal{S}_{q, *}\left(\mathbb{R}_{q}\right) .
$$


- $\mathcal{S}_{\mathrm{q}, *}^{\prime}\left(\mathbb{R}_{\mathrm{q}}\right)$ the space of even q-tempered distributions in $\mathbb{R}_{\mathrm{q}}$. That is the topological dual of $\mathcal{S}_{\mathrm{q}, *}\left(\mathbb{R}_{\mathrm{q}}\right)$.

- $\mathcal{D}_{\mathrm{q}, *}\left(\mathbb{R}_{\mathrm{q}}\right)$ the space of even functions infinitely q-differentiable on $\mathbb{R}_{\mathrm{q}}$ with compact support in $\mathbb{R}_{\mathrm{q}}$. We equip this space with the topology of the uniform convergence of the functions and their q-derivatives.

- $\mathcal{C}_{\mathrm{q}, *, 0}\left(\mathbb{R}_{\mathrm{q}}\right)$ the space of even functions $f$ defined on $\mathbb{R}_{\mathrm{q}}$ continuous on 0 , infinitely q-differentiable and

$$
\lim _{x \rightarrow \infty} f(x)=0, \quad\|f\|_{\mathcal{C}_{\mathrm{q}, *, 0}}=\sup _{x \in \mathbb{R}_{\mathrm{q}}}|f(x)|<+\infty
$$

- $\mathcal{H}_{\mathrm{q}, *}\left(\mathbb{R}_{\mathrm{q}}\right)$ the space of even functions $\mathrm{f}$ defined on $\mathbb{R}_{\mathbf{q}}$ continuous on 0 with compact support such that

$$
\|f\|_{\mathcal{H}_{\mathrm{q}, *}}=\sup _{x \in \mathbb{R}_{\mathrm{q}}}|\mathrm{f}(\mathrm{x})|<+\infty
$$

\section{$2.5 \quad$ q-Bessel function}

The following properties of the normalized q-Bessel function is given (see [13]) by

$$
j_{\alpha}\left(x ; q^{2}\right)=\Gamma_{q^{2}}(\alpha+1) \sum_{k=0}^{\infty} \frac{(-1)^{k} q^{k(k-1)}}{\Gamma_{q^{2}}(k+1) \Gamma_{q^{2}}(\alpha+k+1)}\left(\frac{x}{1+q}\right)^{2 k} .
$$

This function is bounded and for every $x \in \mathbb{R}_{\mathrm{q}}$ and $\alpha>-\frac{1}{2}$ we have

$$
\begin{gathered}
\left|j_{\alpha}\left(x ; q^{2}\right)\right| \leq \frac{1}{\left(q ; q^{2}\right)_{\infty}^{2}} \\
\left(\frac{1}{x} D_{q}\right) j_{\alpha}\left(. ; q^{2}\right)(x)=-\frac{(1-q)}{\left(1-q^{2 \alpha+2}\right)} j_{\alpha+1}\left(q x ; q^{2}\right), \\
\left(\frac{1}{x} D_{q}\right)\left(x^{2 \alpha} j_{\alpha}\left(x ; q^{2}\right)\right)=\frac{1-q^{2 \alpha}}{1-q} x^{2(\alpha-1)} j_{\alpha-1}\left(x ; q^{2}\right), \\
\left|D_{q} j_{\alpha}\left(x ; q^{2}\right)\right| \leq \frac{(1-q)}{\left(1-q^{2 \alpha+2}\right)} \frac{x}{\left(q ; q^{2}\right)_{\infty}^{2}} .
\end{gathered}
$$

We remark that for $\lambda \in \mathbb{C}$, the function $j_{\alpha}\left(\lambda x ; q^{2}\right)$ is the unique solution of the $q$-differential system

$$
\left\{\begin{array}{l}
\Delta_{q, \alpha} U(x, q)=-\lambda^{2} U(x, q), \\
u(0, q)=1 ;\left.D_{q, x} U(x, q)\right|_{x=0}=0,
\end{array}\right.
$$


where $\Delta_{\mathrm{q}, \alpha}$ is the q-Bessel operator defined by

$$
\begin{aligned}
\Delta_{q, \alpha} f(x) & =\frac{1}{\chi^{2 \alpha+1}} D_{q}\left[x^{2 \alpha+1} D_{q} f\right]\left(q^{-1} x\right) \\
& =q^{2 \alpha+1} \Delta_{q} f(x)+\frac{1-q^{2 \alpha+1}}{(1-q) q^{-1} \chi} D_{q} f\left(q^{-1} x\right),
\end{aligned}
$$

where

$$
\Delta_{q} f(x)=\Lambda_{q}^{-1} D_{q}^{2} f(x)=\left(D_{q}^{2} f\right)\left(q^{-1} x\right),
$$

and for $k \in \mathbb{N}$ and $\lambda \in \mathbb{R}_{\mathbf{q},+}$,

$$
\Delta_{\mathbf{q}, x}^{\mathrm{k}} j_{\alpha}\left(\lambda x ; q^{2}\right)=(-1)^{\mathrm{k}} \lambda^{2 \mathrm{k}} j_{\alpha}\left(\lambda x ; q^{2}\right) .
$$

\section{6 q-Bessel Translation operator}

$\mathrm{T}_{\mathbf{q}, x}^{\alpha}, \chi \in \mathbb{R}_{\mathbf{q},+}$ is the $\mathbf{q}$-generalized translation operator associated with the $\mathbf{q}$-Bessel transform is introduced in [13] and rectified in [17, where it is defined by the use of Jackson's q-integral and the q-shifted factorial as

$$
T_{q, x}^{\alpha} f(y)=\int_{0}^{+\infty} f(t) D_{\alpha, q}(x, y, t) t^{2 \alpha+1} d_{q} t, \quad \alpha>-1
$$

with

$$
D_{\alpha, q}(x, y, z)=c_{\alpha, q}^{2} \int_{0}^{+\infty} j_{\alpha}\left(x t ; q^{2}\right) j_{\alpha}\left(y t ; q^{2}\right) j_{\alpha}\left(z t ; q^{2}\right) t^{2 \alpha+1} d_{q} t
$$

where

$$
c_{\alpha, q}=\frac{1}{1-q} \frac{\left(q^{2 \alpha+2} ; q^{2}\right)_{\infty}}{\left(q^{2} ; q^{2}\right)_{\infty}}
$$

In particular the following product formula holds

$$
T_{q, x}^{\alpha} j_{\alpha}\left(y, q^{2}\right)=j_{\alpha}\left(x, q^{2}\right) j_{\alpha}\left(y, q^{2}\right) .
$$

It is shown in [18] that for $f \in \mathrm{L}_{\alpha, q}^{1}\left(\mathbb{R}_{\mathbf{q},+}\right), \quad \mathrm{T}_{\mathbf{q}, \mathrm{f}}^{\alpha} \mathrm{f} \in \mathrm{L}_{\alpha, \mathbf{q}}^{1}\left(\mathbb{R}_{\mathbf{q},+}\right)$ and

$$
\left\|T_{q, x}^{\alpha} f\right\|_{1, \alpha, q}=\|f\|_{1, \alpha, q} .
$$

\subsection{The q-convolution and the q-Bessel Fourier transform}

The q-Bessel Fourier transform $\mathcal{F}_{\alpha, q}$ and the $q$-Bessel convolution product are defined for suitable functions $f, g$ as follows

$$
\mathcal{F}_{\alpha, q}(f)(\lambda)=\int_{0}^{\infty} f(x) j_{\alpha}\left(\lambda x ; q^{2}\right) d_{q} \sigma(x)
$$




$$
f *_{\alpha, q} g(x)=\int_{0}^{+\infty} T_{q, x}^{\alpha} f(y) g(y) d_{q} \sigma(y)
$$

The q-Bessel Fourier transform $\mathcal{F}_{\alpha, \mathrm{q}}$ is a modified version of the q-analogue of the Hankel transform defined in [15].

It is shown in [13, 17, 14, that the q-Bessel Fourier transform $\mathcal{F}_{\alpha, q}$ satisfies the following properties:

Proposition 2.1. If $\mathrm{f} \in \mathrm{L}_{\alpha, \mathbf{q}}^{1}\left(\mathbb{R}_{\mathrm{q},+}\right)$, then $\mathcal{F}_{\alpha, \mathrm{q}}(\mathbf{f}) \in \mathcal{C}_{\mathrm{q}, *, 0}\left(\mathbb{R}_{\mathrm{q},+}\right)$ and

$$
\left\|\mathcal{F}_{\alpha, q}(f)\right\|_{\mathcal{C}_{q, *, o}} \leq B_{\alpha, q}\|f\|_{1, \alpha, q} .
$$

where

$$
B_{\alpha, q}=\frac{1}{(1-q)} \frac{\left(-q^{2} ; q^{2}\right)_{\infty}\left(-q^{2 \alpha+2} ; q^{2}\right)}{\left(q^{2} ; q^{2}\right)_{\infty}}
$$

Proposition 2.2. Given two functions $\mathrm{f}, \mathrm{g} \in \mathrm{L}_{\alpha, \mathrm{q}}^{1}\left(\mathbb{R}_{\mathrm{q},+}\right)$, then

$$
f *_{\alpha, q} g \in L_{\alpha, q}^{1}\left(\mathbb{R}_{q,+}\right),
$$

and

$$
\mathcal{F}_{\alpha, \mathrm{q}}\left(\mathrm{f} *_{\alpha, \mathrm{q}} \mathrm{g}\right)=\mathcal{F}_{\alpha, \mathrm{q}}(\mathrm{f}) \mathcal{F}_{\alpha, \mathrm{q}}(\mathrm{g}) .
$$

Theorem 2.3. (Inversion formula)

1. If $\mathrm{f} \in \mathrm{L}_{\alpha, \mathbf{q}}^{1}\left(\mathbb{R}_{\mathbf{q},+}\right)$ such that $\mathcal{F}_{\alpha, \mathbf{q}}(\mathrm{f}) \in \mathrm{L}_{\alpha, \mathbf{q}}^{1}\left(\mathbb{R}_{\mathbf{q},+}\right)$, then for all $\mathrm{x} \in \mathbb{R}_{\mathrm{q},+}$, we have

$$
f(x)=\int_{0}^{\infty} \mathcal{F}_{\alpha, q}(f)(y) j_{\alpha}\left(x y ; q^{2}\right) d_{q} \sigma(y) .
$$

2. $\mathcal{F}_{\alpha, \mathrm{q}}(\mathrm{f})$ is an isomorphism of $\mathcal{S}_{*, \mathrm{q}}\left(\mathbb{R}_{\mathrm{q}}\right)$ and $\mathcal{F}_{\alpha, \mathrm{q}}^{2}(\mathrm{f})=\mathrm{Id}$.

- Note that the inversion formula is valid for $f \in \mathrm{L}_{\alpha, \mathbf{q}}^{1}\left(\mathbb{R}_{\mathbf{q},+}\right)$ without the additional condition $\mathcal{F}_{\alpha, \mathrm{q}}(\mathrm{f}) \in \mathrm{L}_{\alpha, \mathrm{q}}^{1}\left(\mathbb{R}_{\mathrm{q},+}\right)$.

$\mathcal{F}_{\alpha, \mathbf{q}}(\mathrm{f})$ can be extended to $\mathrm{L}_{\alpha, \mathbf{q}}^{2}\left(\mathbb{R}_{\mathbf{q},+}\right)$ and we have the following theorem:

Theorem 2.4. (q-Plancherel theorem)

$\mathcal{F}_{\alpha, \mathbf{q}}(\mathrm{f})$ is an isomorphism of $\mathrm{L}_{\alpha, \mathbf{q}}^{2}\left(\mathbb{R}_{\mathbf{q},+}\right)$, we have $\left\|\mathcal{F}_{\alpha, \mathbf{q}}(\mathrm{f})\right\|_{2, \alpha, q}=\|f\|_{2, \alpha, q}$, for $\mathrm{f} \in \mathrm{L}_{\alpha, \mathbf{q}}^{2}\left(\mathbb{R}_{\mathbf{q},+}\right)$ and $\mathcal{F}_{\alpha, q}^{-1}(f)=\mathcal{F}_{\alpha, q}(f)$.

\section{Proposition 2.5.}

(i) For $\mathrm{f} \in \mathrm{L}_{\alpha, \mathbf{q}}^{\mathrm{p}}\left(\mathbb{R}_{\mathbf{q},+}\right)$, $\mathrm{p} \in\left[1, \infty\left[, \mathrm{g} \in \mathrm{L}_{\alpha, \mathbf{q}}^{1}\left(\mathbb{R}_{\mathbf{q},+}\right)\right.\right.$ we have $\mathrm{f} *_{\alpha, \mathbf{q}} \mathrm{g} \in \mathrm{L}_{\alpha, \mathbf{q}}^{\mathrm{p}}\left(\mathbb{R}_{\mathbf{q},+}\right)$ and $\left\|f *_{\alpha q} g\right\|_{p, \alpha, q} \leq\|f\|_{p, \alpha, q}\|g\|_{1, \alpha, q}$.

(ii) $\int_{0}^{\infty} \mathcal{F}_{\alpha, \mathbf{q}}(f)(\xi) g(\xi) d_{\mathbf{q}} \sigma(\xi)=\int_{0}^{\infty} f(\xi) \mathcal{F}_{\alpha, \mathbf{q}}(g)(\xi) d_{\mathbf{q}} \sigma(\xi) ; \quad f, g \in L_{\alpha, \mathbf{q}}^{1}\left(\mathbb{R}_{\mathbf{q},+}\right)$. 
(iii) $\mathcal{F}_{\alpha, q}\left(T_{q, x}^{\alpha} f\right)(\xi)=j_{\alpha}\left(\xi x ; q^{2}\right) \mathcal{F}_{\alpha, q}(f)(\xi) ; \quad f \in L_{\alpha, q}^{1}\left(\mathbb{R}_{q,+}\right)$.

Specially, we choose $q \in\left[0, q_{0}\right]$ where $q_{0}$ is the first zero of the function [17: $\mathrm{q} \mapsto{ }_{1} \phi_{1}(0, \mathrm{q}, \mathrm{q} ; \mathrm{q})$ under the condition $\frac{\log (1-\mathrm{q})}{\log \mathrm{q}} \in \mathbb{Z}$.

Definition 2.6. [11, 9] A bounded complex even measure $\mu$ on $\mathbb{R}_{\mathrm{q}}$ is a bounded linear functional $\mu$ on $\mathcal{H}_{\mathrm{q}, *}\left(\mathbb{R}_{\mathrm{q}}\right)$, i.e., for all $\mathrm{f}$ in $\mathcal{H}_{\mathrm{q}, *}\left(\mathbb{R}_{\mathrm{q}}\right)$, we have

$$
|\mu(f)| \leq C\|f\|_{\mathcal{H}_{q, *}},
$$

where $\mathrm{C}>0$ is a positive constant.

Denote the space of all such measure by $\mathcal{M}^{\prime}\left(\mathbb{R}_{\mathbf{q},+}\right)$.

Note that $\mu \in \mathcal{M}^{\prime}\left(\mathbb{R}_{\mathbf{q},+}\right)$ can be identified with a function $\widetilde{\mu}$ on $\widetilde{\mathbb{R}}_{\mathbf{q},+}$ such that $\widetilde{\mu}$ restricted to $\mathbb{R}_{\mathbf{q},+}$ is $\mathrm{L}_{\alpha, \mathbf{q}}^{1}\left(\mathbb{R}_{\mathbf{q},+}\right)$ :

$$
\mu(f)=\mu(\{0\}) f(0)+\int_{0}^{\infty} \widetilde{\mu}(x) f(x) d_{q}(x), \quad\left(f \in \mathcal{H}_{q, *}\left(\mathbb{R}_{q}\right)\right) .
$$

For $\mu \in \mathcal{M}^{\prime}\left(\mathbb{R}_{\mathbf{q},+}\right)$ denote $\|\mu\|=|\mu|\left(\mathbb{R}_{\mathbf{q},+}\right)$ where $|\mu|$ is the absolute value of $\mu$.

Definition 2.7. The q-Bessel Fourier transform of a measure $\mu$ in $\mathcal{M}^{\prime}\left(\mathbb{R}_{\mathbf{q},+}\right)$ is defined for all $\varphi \in \mathcal{S}_{\mathbf{q}, *}\left(\mathbb{R}_{\mathbf{q}}\right)$ by

$$
\mathcal{F}_{\alpha, q} \mu(\lambda)=b_{\alpha, q} \int_{0}^{+\infty} j_{\alpha}\left(\lambda x ; q^{2}\right) d \mu(x) .
$$

The $\mathbf{q}$-Bessel convolution product of a measure $\mu \in \mathcal{M}^{\prime}\left(\mathbb{R}_{\mathbf{q},+}\right)$ and a suitable function $\mathbf{f}$ on $\mathbb{R}_{\mathbf{q},+}$ is defined by

$$
\mu *_{\alpha, q} f(x)=\int_{0}^{\infty} T_{q, x}^{\alpha} f(y) d \mu(y) .
$$

Proposition 2.8. (1) The $\mathbf{q}$-Bessel Fourier transform $\mathcal{F}_{\alpha, \mathbf{q}}$ of a measure $\mu$ in $\mathcal{M}^{\prime}\left(\mathbb{R}_{\mathbf{q},+}\right)$ is the $\mathrm{q}$-tempered distribution $\mathcal{F}_{\alpha, \mathrm{q}} \mu$ given by:

$$
\left\langle\mathcal{F}_{\alpha, q} \mu, \varphi\right\rangle=\left\langle\mu, \mathcal{F}_{\alpha, q} \varphi\right\rangle=\int_{0}^{+\infty} \mathcal{F}_{\alpha, q} \varphi(\lambda) d_{q} \mu(\lambda) .
$$

(2) For all $x, \lambda \in \mathbb{R}_{\mathbf{q},+}$ we have

$$
\mathrm{T}_{\mathrm{q}, x}^{\alpha} \mathcal{F}_{\alpha, \mathrm{q}} \mu(\lambda)=\mathrm{b}_{\alpha, \mathrm{q}} \int_{0}^{+\infty} j_{\alpha}\left(x \mathrm{t} ; \mathrm{q}^{2}\right) j_{\alpha}\left(\lambda \mathrm{t} ; \mathrm{q}^{2}\right) \mathrm{d}_{\mathrm{q}} \mu(\mathrm{t}) .
$$

(3) For all $\mu \in \mathcal{M}^{\prime}\left(\mathbb{R}_{\mathbf{q},+}\right), \mathcal{F}_{\alpha, \mathbf{q}} \mu$ is continuous on $\mathbb{R}_{\mathbf{q},+}$, and

$$
\lim _{\lambda \rightarrow \infty} \mathcal{F}_{\alpha, q} \mu(\lambda)=\mu(\{0\}) .
$$

$\mathcal{F}_{\alpha, \mathrm{q}}$ maps one to one $\mathcal{M}^{\prime}\left(\mathbb{R}_{\mathrm{q},+}\right)$ into $\mathcal{C}_{\mathrm{b}}\left(\mathbb{R}_{\mathrm{q},+}\right)$, (the space of continuous and bounded functions on $\mathbb{R}_{\mathrm{q},+}$ ). 
(4) If $\mu \in \mathcal{M}^{\prime}\left(\mathbb{R}_{\mathbf{q},+}\right)$ and $f \in \mathrm{L}_{\alpha, \mathbf{q}}^{\mathrm{p}}\left(\mathbb{R}_{\mathrm{q},+}\right), \mathrm{p}=1,2$ then $\mu *_{\alpha, \mathrm{q}} f \in \mathrm{L}_{\alpha, \mathbf{q}}^{\mathrm{p}}\left(\mathbb{R}_{\mathbf{q},+}\right)$ and

$$
\left\|\mu *_{\alpha, q} f\right\|_{p, \alpha, q} \leq\|\mu\|\|f\|_{p, \alpha, q} .
$$

(5) For all $\mu \in \mathcal{M}^{\prime}\left(\mathbb{R}_{\mathbf{q},+}\right)$ and $f \in \mathrm{L}_{\alpha, \mathbf{q}}^{\mathrm{p}}\left(\mathbb{R}_{\mathbf{q},+}\right), \mathrm{p}=1,2$ we have

$$
\mathcal{F}_{\alpha, q}\left(\mu *_{\alpha, q} f\right)=\mathcal{F}_{\alpha, q}(\mu) \mathcal{F}_{\alpha, q}(f) .
$$

Definition 2.9. Let $\mu \in \mathcal{M}^{\prime}\left(\mathbb{R}_{\mathbf{q},+}\right)$ and $\mathrm{a}>0$. We define the $\mathbf{q}$-dilated measure $\mu_{\mathrm{a}}$ of $\mu$ by

$$
\int_{0}^{\infty} \varphi(x) d_{\mathrm{q}} \mu_{\mathrm{a}}(x)=\int_{0}^{\infty} \varphi(\mathrm{ax}) \mathrm{d}_{\mathrm{q}} \mu(x), \quad \varphi \in \mathcal{H}_{\mathrm{q}, *}\left(\mathbb{R}_{\mathrm{q}}\right) .
$$

Proposition 2.10. (i) When $\mu=f(x) x^{2 \alpha+1} d_{q} x$, with $f \in L_{\alpha, q}^{1}\left(\mathbb{R}_{\mathfrak{q},+}\right)$, the measure $\mu_{a}$, $a>0$, is given by the function

$$
f_{a}(x)=\frac{1}{a^{2 \alpha+2}} f\left(\frac{x}{a}\right), \quad x \geq 0 .
$$

(ii) Let $\mu \in \mathcal{M}^{\prime}\left(\mathbb{R}_{\mathbf{q},+}\right)$, then

$$
\mathcal{F}_{\alpha, \mathfrak{q}}\left(\mu_{\mathrm{a}}\right)(\lambda)=\mathcal{F}_{\alpha, \mathfrak{q}}(\mu)(\mathrm{a} \lambda), \text { for all } \quad \lambda \geq 0
$$

(iii) For $\mu \in \mathcal{M}^{\prime}\left(\mathbb{R}_{\mathrm{q},+}\right)$ and $\mathrm{f} \in \mathrm{L}_{\alpha, \mathrm{q}}^{\mathrm{p}}\left(\mathbb{R}_{\mathrm{q},+}\right), \mathrm{p}=1,2$ we have

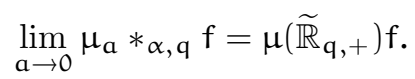

where the limit is in $\mathrm{L}_{\alpha, \mathbf{q}}^{\mathrm{p}}\left(\mathbb{R}_{\mathbf{q},+}\right)$.

(iv) Let $\mathrm{g} \in \mathrm{L}_{\alpha, \mathbf{q}}^{1}\left(\mathbb{R}_{\mathbf{q},+}\right)$ and $\mathrm{f} \in \mathrm{L}_{\alpha, \mathbf{q}}^{\mathrm{p}}\left(\mathbb{R}_{\mathbf{q},+}\right), 1<\mathrm{p}<\infty$. Then

$$
\lim _{a \rightarrow \infty} f *_{\alpha, q} g_{a}=0
$$

where the limit is in $\mathrm{L}_{\alpha, \mathbf{q}}^{\mathrm{p}}\left(\mathbb{R}_{\mathbf{q},+}\right)$.

Proof. Statement of (i) and (ii) are obvious. A standard argument gives (iii). Let us verify (iv). If $f, g \in \mathcal{D}_{\mathrm{q}, *}\left(\mathbb{R}_{\mathrm{q}}\right)$ then by (58) and (61) we have

$$
\begin{aligned}
\left\|f *_{\alpha, q} g_{a}\right\|_{p, \alpha, q} & \leq\|f\|_{1, \alpha, q}\left\|g_{a}\right\|_{p, \alpha, q} \\
& =a^{\frac{-2(\alpha+1)(p-1)}{p}}\|f\|_{1, \alpha, q}\|g\|_{p, \alpha, q} \rightarrow 0, \quad \text { as } \quad a \rightarrow \infty .
\end{aligned}
$$

For arbitrary $g \in \mathrm{L}_{\alpha, \mathbf{q}}^{1}\left(\mathbb{R}_{\mathbf{q},+}\right)$ and $f \in \mathrm{L}_{\alpha, \mathbf{q}}^{\mathrm{p}}\left(\mathbb{R}_{\mathbf{q},+}\right)$ the result follows by density.

Given a measure $\mu \in \mathcal{M}^{\prime}\left(\mathbb{R}_{\mathrm{q},+}\right)$. Denote

$$
c_{\mu, \alpha, q}=\int_{0}^{\infty} \mathcal{F}_{\alpha, q}(\mu)(\lambda) \frac{\mathrm{d}_{\mathrm{q}} \lambda}{\lambda} .
$$




\section{3 q-Calderón's formula for functions}

In this section, we establish the q-Calderón's reproducing identity for functions using the properties of q-Fourier Bessel transform $\mathcal{F}_{\alpha, \mathrm{q}}$ and q-Bessel convolution $*_{\alpha, \mathrm{q}}$.

Theorem 3.1. Let $\varphi$ and $\psi \in \mathrm{L}_{\alpha, \mathbf{q}}^{1}\left(\mathbb{R}_{\mathbf{q},+}\right)$ be such that following admissibility condition holds

$$
\int_{0}^{\infty} \mathcal{F}_{\alpha, q}(\varphi)(\xi) \mathcal{F}_{\alpha, q}(\psi)(\xi) \frac{d_{q} \xi}{\xi}=1
$$

then for all $\mathrm{f} \in \mathrm{L}_{\alpha, \mathbf{q}}^{1}\left(\mathbb{R}_{\mathbf{q},+}\right)$, the following Calderón's reproducing identity holds:

$$
f(x)=\int_{0}^{\infty}\left(f *_{\alpha, q} \varphi_{t} *_{\alpha, q} \psi_{t}\right)(x) \frac{d_{q} t}{t} .
$$

Proof. Taking q-Bessel Fourier transform of the right-hand side of (67), we get

$$
\begin{aligned}
\mathcal{F}_{\alpha, \mathrm{q}}\left[\int_{0}^{\infty}\left(\mathrm{f} *_{\alpha, \mathrm{q}} \varphi_{\mathrm{t}} *_{\alpha, \mathrm{q}} \psi_{\mathrm{t}}\right)(\mathrm{x}) \frac{\mathrm{d}_{\mathrm{q}} \mathrm{t}}{\mathrm{t}}\right](\xi) & =\int_{0}^{\infty} \mathcal{F}_{\alpha, \mathrm{q}}(\mathrm{f})(\xi) \mathcal{F}_{\alpha, \mathrm{q}}\left(\varphi_{\mathrm{t}}\right)(\xi) \mathcal{F}_{\alpha, \mathrm{q}}\left(\psi_{\mathrm{t}}\right)(\xi) \frac{\mathrm{d}_{\mathrm{q}} \mathrm{t}}{\mathrm{t}} \\
& =\mathcal{F}_{\alpha, \mathrm{q}}(\mathrm{f})(\xi) \int_{0}^{\infty} \mathcal{F}_{\alpha, \mathrm{q}}\left(\varphi_{\mathrm{t}}\right)(\xi) \mathcal{F}_{\alpha, \mathrm{q}}\left(\psi_{\mathrm{t}}\right)(\xi) \frac{\mathrm{d}_{\mathrm{q}} \mathrm{t}}{\mathrm{t}} \\
& =\mathcal{F}_{\alpha, \mathrm{q}}(\mathrm{f})(\xi) \int_{0}^{\infty} \mathcal{F}_{\alpha, \mathrm{q}}(\varphi)(\mathrm{t} \xi) \mathcal{F}_{\alpha, \mathrm{q}}(\psi)(\mathrm{t} \xi) \frac{\mathrm{d}_{\mathrm{q}} \mathrm{t}}{\mathrm{t}} \\
& =\mathcal{F}_{\alpha, \mathrm{q}}(\mathrm{f})(\xi) .
\end{aligned}
$$

Now, by putting $t \xi=s$, we get

$$
\int_{0}^{\infty} \mathcal{F}_{\alpha, q}(\varphi)(\mathrm{t} \xi) \mathcal{F}_{\alpha, q}(\psi)(\mathrm{t} \xi) \frac{\mathrm{d}_{\mathrm{q}} \mathrm{t}}{\mathrm{t}}=\int_{0}^{\infty} \mathcal{F}_{\alpha, q}(\varphi)(\mathrm{s}) \mathcal{F}_{\alpha, q}(\psi)(\mathrm{s}) \frac{\mathrm{d}_{\mathrm{q}} \mathrm{s}}{\mathrm{s}}=1 .
$$

Hence, the result follows.

The equality (67) can be interpreted in the following $\mathrm{L}^{2}$-sense.

Theorem 3.2. Suppose $\varphi \in \mathrm{L}_{\alpha, \mathbf{q}}^{1}\left(\mathbb{R}_{\mathbf{q},+}\right)$ and satisfies

$$
\int_{0}^{\infty}\left[\mathcal{F}_{\alpha, q}(\varphi)(\xi)\right]^{2} \frac{\mathrm{d}_{\mathrm{q}} \xi}{\xi}=1 .
$$

For $\mathrm{f} \in \mathrm{L}_{\alpha, \mathbf{q}}^{1}\left(\mathbb{R}_{\mathbf{q},+}\right) \cap \mathrm{L}_{\alpha, \mathbf{q}}^{2}\left(\mathbb{R}_{\mathbf{q},+}\right)$, suppose that

$$
\left.f^{\varepsilon, \delta}(x)=\int_{\varepsilon}^{\delta}(f * \alpha, q) \varphi_{t} *_{\alpha, q} \varphi_{t}\right)(x) \frac{d_{q} t}{t}
$$

then

$$
\left\|f^{\varepsilon, \delta}-\mathrm{f}\right\|_{2, \alpha, q} \longrightarrow 0 \quad \text { as } \varepsilon \rightarrow 0 \text { and } \delta \rightarrow \infty \text {. }
$$


Proof. Taking q-Bessel Fourier transform of both sides of (69) and using Fubini's theorem, we get

$$
\mathcal{F}_{\alpha, q}\left(f^{\varepsilon, \delta}\right)(\xi)=\mathcal{F}_{\alpha, q}(f)(\xi) \int_{\varepsilon}^{\delta}\left[\mathcal{F}_{\alpha, q}(\varphi)(t \xi)\right]^{2} \frac{d_{q} t}{t}
$$

by Proposition 2.5, we have

$$
\begin{aligned}
\left\|\varphi_{\mathrm{t}} *_{\alpha, \mathrm{q}} \varphi_{\mathrm{t}} *_{\alpha, \mathrm{q}} \mathrm{f}\right\|_{2, \alpha, \mathrm{q}} & \leq\left\|\varphi_{\mathrm{t}} *_{\alpha, \mathrm{q}} \varphi_{\mathrm{t}}\right\|_{1, \alpha, \mathrm{q}}\|\mathrm{f}\|_{2, \alpha, \mathrm{q}} \\
& \leq\left\|\varphi_{\mathrm{t}}\right\|_{1, \alpha, q}^{2}\|f\|_{2, \alpha, q} .
\end{aligned}
$$

Now using above inequality, Minkowski's inequality and relation (29), we get

$$
\begin{aligned}
& \left\|f^{\varepsilon, \delta}\right\|_{2, \alpha, q}^{2}=\int_{0}^{\infty}\left|\int_{\varepsilon}^{\delta}\left(\varphi_{t} *_{\alpha, q} \varphi_{t} *_{\alpha, q} f\right)(x) \frac{d_{q} t}{t}\right|^{2} d_{q} \sigma(x) \\
& \leq \int_{\varepsilon}^{\delta} \int_{0}^{\infty}\left|\left(\varphi_{\mathrm{t}} *_{\alpha, q} \varphi_{\mathrm{t}} *_{\alpha, q} f\right)(x)\right|^{2} \mathrm{~d}_{\mathrm{q}} \sigma(x) \frac{\mathrm{d}_{\mathrm{q}} \mathrm{t}}{\mathrm{t}} \\
& \leq \int_{\varepsilon}^{\delta}\left\|\varphi_{\mathrm{t}} *_{\alpha, q} \varphi_{\mathrm{t}} *_{\alpha, q} f\right\|_{2, \alpha, q} \frac{\mathrm{d}_{\mathrm{q}} \mathrm{t}}{\mathrm{t}} \\
& \leq\left\|\varphi_{\mathrm{t}}\right\|_{1, \alpha, \mathrm{q}}^{2}\|\mathrm{f}\|_{2, \alpha, \mathrm{q}} \int_{\varepsilon}^{\delta} \frac{\mathrm{d}_{\mathrm{q}} \mathrm{t}}{\mathrm{t}} \\
& =\left\|\varphi_{\mathrm{t}}\right\|_{1, \alpha, q}^{2}\|f\|_{2, \alpha, q} \log _{\mathrm{q}}\left(\frac{\delta}{\varepsilon}\right) .
\end{aligned}
$$

Hence, by Theorem 2.4, we get

$$
\begin{array}{ll}
\lim _{\substack{\varepsilon \rightarrow 0 \\
\delta \rightarrow \infty}}\left\|f^{\varepsilon, \delta}-f\right\|_{2, \alpha, q}^{2}= & \lim _{\substack{\varepsilon \rightarrow 0 \\
\delta \rightarrow \infty}}\left\|\mathcal{F}_{\alpha, q}\left(f^{\varepsilon, \delta}\right)-\mathcal{F}_{\alpha, q}(f)\right\|_{2, \alpha, q}^{2} \\
& =\lim _{\substack{\varepsilon \rightarrow 0 \\
\delta \rightarrow \infty}} \int_{0}^{\infty}\left|\mathcal{F}_{\alpha, q}(f)(\xi)\left(1-\int_{\varepsilon}^{\delta}\left[\mathcal{F}_{\alpha, q}(\varphi)(t \xi)\right]^{2} \frac{d_{q} t}{t}\right)\right|^{2} d_{q} \sigma(x)=0 .
\end{array}
$$

Since $\left|\mathcal{F}_{\alpha, q}(f)(\xi)\left(1-\int_{\varepsilon}^{\delta}\left[\mathcal{F}_{\alpha, q}(\varphi)(t \xi)\right]^{2} \frac{d_{q} t}{t}\right)\right| \leq\left|\mathcal{F}_{\alpha, q}(f)(\xi)\right|$, therefore, by the dominated convergence theorem, the result follows.

The reproducing identity (67) holds in the pointwise sense under different sets of nice conditions.

Theorem 3.3. Suppose $\mathrm{f}, \mathcal{F}_{\alpha, \mathrm{q}} \mathrm{f} \in \mathrm{L}_{\alpha, \mathrm{q}}^{1}\left(\mathbb{R}_{\mathbf{q},+}\right)$. Let $\varphi \in \mathrm{L}_{\alpha, \mathrm{q}}^{1}\left(\mathbb{R}_{\mathbf{q},+}\right)$ and satisfies

$$
\int_{0}^{\infty}\left[\mathcal{F}_{\alpha, q} \varphi(\mathrm{t} \xi)\right]^{2} \frac{\mathrm{d}_{\mathrm{q}} \mathrm{t}}{\mathrm{t}}=1
$$


then

$$
\lim _{\substack{\varepsilon \rightarrow 0 \\ \delta \rightarrow \infty}} f^{\varepsilon, \delta}(x)=f(x)
$$

where $\mathrm{f}^{\varepsilon, \delta}$ is given by (69).

Proof. by Proposition 2.5, we have

$$
\left\|\varphi_{\mathrm{t}} *_{\alpha, q} \varphi_{\mathrm{t}} *_{\alpha, q} f\right\|_{1, \alpha, q} \leq\left\|\varphi_{\mathrm{t}}\right\|_{1, \alpha, q}^{2}\|f\|_{1, \alpha, q} .
$$

Now

$$
\begin{aligned}
\left\|f^{\varepsilon, \delta}\right\|_{1, \alpha, q} & =\int_{0}^{\infty}\left|\int_{\varepsilon}^{\delta}\left(\varphi_{\mathrm{t}} *_{\alpha, q} \varphi_{\mathrm{t}} *_{\alpha, q} f\right)(x) \frac{\mathrm{d}_{\mathrm{q}} \mathrm{t}}{\mathrm{t}}\right| \mathrm{d}_{\mathrm{q}} \sigma(x) \\
& \leq \int_{\varepsilon}^{\delta} \int_{0}^{\infty}\left|\left(\varphi_{\mathrm{t}} *_{\alpha, \mathrm{q}} \varphi_{\mathrm{t}} *_{\alpha, \mathrm{q}} f\right)(x)\right| \mathrm{d}_{\mathrm{q}} \sigma(x) \frac{\mathrm{d}_{\mathrm{q}} \mathrm{t}}{\mathrm{t}} \\
& \leq \int_{\varepsilon}^{\delta}\left\|\varphi_{\mathrm{t}} *_{\alpha, \mathrm{q}} \varphi_{\mathrm{t}} *_{\alpha, \mathrm{q}} \mathrm{f}\right\|_{1, \alpha, \mathrm{q}} \frac{\mathrm{d}_{\mathrm{q}} \mathrm{t}}{\mathrm{t}} \\
& \leq\left\|\varphi_{\mathrm{t}}\right\|_{1, \alpha, \mathrm{q}}^{2}\|\mathrm{f}\|_{1, \alpha, q} \log _{\mathrm{q}}\left(\frac{\delta}{\varepsilon}\right) .
\end{aligned}
$$

Therefore, $f^{\varepsilon, \delta} \in \mathrm{L}_{\alpha, \mathbf{q}}^{1}\left(\mathbb{R}_{\mathbf{q},+}\right)$. Also using Fubini's theorem and taking q- Bessel Fourier transform of $f^{\varepsilon, \delta}$, we get

$$
\begin{aligned}
\mathcal{F}_{\alpha, q} f^{\varepsilon, \delta}(\xi) & =\int_{0}^{\infty} j_{\alpha}\left(x \xi ; q^{2}\right)\left(\int_{\varepsilon}^{\delta}\left(\varphi_{\mathrm{t}} *_{\alpha, q} \varphi_{\mathrm{t}} *_{\alpha, \mathrm{q}} f\right)(x) \frac{\mathrm{d}_{\mathrm{q}} \mathrm{t}}{\mathrm{t}}\right) \mathrm{d}_{\mathrm{q}} \sigma(x) \\
& =\int_{\varepsilon}^{\delta} \int_{0}^{\infty} j_{\alpha}\left(x \xi ; q^{2}\right)\left(\varphi_{\mathrm{t}} *_{\alpha, q} \varphi_{\mathrm{t}} *_{\alpha, q} f\right)(x) \mathrm{d}_{\mathrm{q}} \sigma(x) \frac{\mathrm{d}_{\mathrm{q}} \mathrm{t}}{\mathrm{t}} \\
& =\int_{\varepsilon}^{\delta} \mathcal{F}_{\alpha, \mathrm{q}} \varphi_{\mathrm{t}}(\xi) \mathcal{F}_{\alpha, \mathrm{q}} \varphi_{\mathrm{t}}(\xi) \mathcal{F}_{\alpha, \mathrm{q}} \mathrm{f}(\xi) \frac{\mathrm{d}_{\mathrm{q}} \mathrm{t}}{\mathrm{t}} \\
& =\mathcal{F}_{\alpha, \mathrm{q}} \mathrm{f}(\xi) \int_{\varepsilon}^{\delta}\left[\mathcal{F}_{\alpha, \mathrm{q}} \varphi(\mathrm{t} \xi)\right]^{2} \frac{\mathrm{d}_{\mathrm{q}} \mathrm{t}}{\mathrm{t}} .
\end{aligned}
$$

Therefore by (171),$\left|\mathcal{F}_{\alpha, q} f^{\varepsilon, \delta}(\xi)\right| \leq\left|\mathcal{F}_{\alpha, q} f(\xi)\right|$. It follows that $\mathcal{F}_{\alpha, q} f^{\varepsilon, \delta} \in \mathrm{L}_{\alpha, q}^{1}\left(\mathbb{R}_{q,+}\right)$. By inversion, we have

$$
f(x)-f^{\varepsilon, \delta}(x)=\int_{0}^{\infty} j_{\alpha}\left(x \xi ; q^{2}\right)\left[\mathcal{F}_{q} f(\xi)-\mathcal{F}_{\alpha, q} f^{\varepsilon, \delta}(\xi)\right] d_{q} \sigma(\xi) .
$$

Putting

$$
\begin{aligned}
g^{\varepsilon, \delta}(x, \xi) & =j_{\alpha}\left(x \xi ; q^{2}\right)\left[\mathcal{F}_{\alpha, q} f(\xi)-\mathcal{F}_{\alpha, q} f^{\varepsilon, \delta}(\xi)\right] \\
& =j_{\alpha}\left(x \xi ; q^{2}\right) \mathcal{F}_{q} f(\xi)\left[1-\int_{\varepsilon}^{\delta}\left[\mathcal{F}_{\alpha, q} \varphi(t \xi)\right]^{2} \frac{d_{q} t}{t}\right],
\end{aligned}
$$


we get

$$
\begin{aligned}
f(x)-f^{\varepsilon, \delta}(x) & =\int_{0}^{\infty} j_{\alpha}\left(x \xi ; q^{2}\right)\left[\mathcal{F}_{\alpha, q} f(\xi)-\mathcal{F}_{\alpha, q} f^{\varepsilon, \delta}(\xi)\right] d_{q} \sigma(\xi) \\
& =\int_{0}^{\infty} g^{\varepsilon, \delta}(x, \xi) d_{q} \sigma(\xi) .
\end{aligned}
$$

Now using (71) and (74), we get

$$
\lim _{\substack{\varepsilon \rightarrow 0 \\ \delta \rightarrow \infty}} g^{\varepsilon, \delta}(x, \xi)=0 .
$$

Since $\left|g^{\varepsilon, \delta}(x, \xi)\right| \leq \frac{1}{\left(q ; q^{2}\right)_{\infty}^{2}}\left|\mathcal{F}_{\alpha, q} f(\xi)\right|$, the dominated convergence theorem yields the result.

\section{4 q-Calderón's formula for finite measures}

It is now possible to define analogues to (2) for the q-Bessel convolution $*_{\alpha, q}$ and investigate its convergence in the $\mathrm{L}_{\alpha, \mathbf{q}}^{2}\left(\mathbb{R}_{\mathbf{q},+}\right) \mathbf{q}$-norm. To this end we need some technical lemmas

Lemma 4.1. Let $\mu \in \mathcal{M}^{\prime}\left(\mathbb{R}_{\mathbf{q},+}\right)$, for $0<\varepsilon<\delta<\infty$ define

$$
\mathrm{G}_{\varepsilon, \delta}\left(x ; q^{2}\right)=\frac{\mu\left(\left[\frac{x}{\delta}, \frac{x}{\varepsilon}\right]\right)}{x^{2 \alpha+2}}, \quad x>0
$$

and

$$
\mathrm{K}_{\varepsilon, \delta}\left(\lambda ; \mathrm{q}^{2}\right)=\int_{\varepsilon}^{\delta} \mathcal{F}_{\alpha, \mathrm{q}}(\mu)(\mathrm{qa} \lambda) \frac{\mathrm{d}_{\mathrm{q}} \mathrm{a}}{\mathrm{a}}, \lambda \geq 0 .
$$

Then $\mathrm{G}_{\varepsilon, \delta} \in \mathrm{L}_{\alpha, \mathbf{q}}^{1}\left(\mathbb{R}_{\mathbf{q},+}\right)$ and

$$
\mathcal{F}_{\alpha, q}\left(G_{\varepsilon, \delta}\right)\left(\lambda ; q^{2}\right)=K_{\varepsilon, \delta}\left(\lambda ; q^{2}\right)-\mu(\{0\}) \log _{q}\left(\frac{\delta}{\varepsilon}\right),
$$

where $\log _{\mathrm{q}}$ is given by (25).

Proof. We have by (25) and (29),

$$
\begin{aligned}
\left|\int_{0}^{\infty} G_{\varepsilon, \delta}\left(x ; q^{2}\right) x^{2 \alpha+1} d_{q} x\right| & \leq \int_{0}^{\infty}\left(\int_{\frac{x}{\delta}}^{\frac{x}{\varepsilon}} d_{q}|\mu|(y)\right) \frac{d_{q} x}{x} \\
& =\int_{0}^{\infty}\left[\int_{0}^{\frac{x}{\varepsilon}} d_{q}|\mu|(y)-\int_{0}^{\frac{x}{\delta}} d_{q}|\mu|(y)\right] \frac{d_{q} x}{x} \\
& =\int_{0}^{\infty}\left[\int_{q \varepsilon y}^{\infty} \frac{d_{q} x}{x}-\int_{q \delta y}^{\infty} \frac{d_{q} x}{x}\right] d_{q}|\mu|(y) \\
& =\int_{0}^{\infty} \log _{q}\left(\frac{\varepsilon}{\delta}\right) d_{q}|\mu|(y) \\
& =|\mu|\left(\widetilde{\mathbb{R}}_{q,+}\right) \log _{q}\left(\frac{\varepsilon}{\delta}\right)<\infty .
\end{aligned}
$$


Using again relation (29) and q-Fubini's theorem we obtain

$$
\begin{aligned}
\mathcal{F}_{\alpha, q}\left(G_{\varepsilon, \delta}\right)(\lambda) & =\int_{0}^{\infty} \int_{\frac{x}{\delta}}^{\frac{x}{\varepsilon}} d_{q} \mu(y) j_{\alpha}\left(\lambda x ; q^{2}\right) \frac{d_{q} x}{x} \\
& =\int_{0}^{\infty} \int_{q \varepsilon y}^{q \delta y} j_{\alpha}\left(\lambda x ; q^{2}\right) \frac{d_{q} x}{x} d_{q} \mu(y) \\
& =\int_{0}^{\infty} \int_{q \varepsilon}^{q \delta} j_{\alpha}\left(\lambda x y ; q^{2}\right) \frac{d_{q} x}{x} d_{q} \mu(y) \\
& =\int_{q \varepsilon}^{q \delta} \int_{0}^{\infty} j_{\alpha}\left(\lambda x y ; q^{2}\right) d_{q} \mu(y) \frac{d_{q} x}{x} \\
& =\int_{q \varepsilon}^{q \delta} \mathcal{F}_{\alpha, q} \mu(\lambda x)-\mu(\{0\}) \frac{d_{q} x}{x} \\
& =\int_{\varepsilon}^{\delta} \mathcal{F}_{\alpha, q} \mu(q \lambda x)-\mu(\{0\}) \frac{d_{q} x}{x} \\
& =K_{\varepsilon, \delta}\left(\lambda ; q^{2}\right)-\mu(\{0\}) \log _{q}\left(\frac{\delta}{\varepsilon}\right) .
\end{aligned}
$$

Lemma 4.2. Let $\mu \in \mathcal{M}^{\prime}\left(\mathbb{R}_{\mathbf{q},+}\right)$, then for $\mathrm{f} \in \mathrm{L}_{\alpha, \mathbf{q}}^{\mathrm{p}}\left(\mathbb{R}_{\mathbf{q},+}\right), \mathrm{p}=1,2$ and $0<\varepsilon<\delta<\infty$, the function

$$
f^{\varepsilon, \delta}\left(x ; q^{2}\right)=\int_{\varepsilon}^{\delta} f *_{\alpha, q} \mu_{a}\left(x ; q^{2}\right) \frac{d_{q} a}{a}
$$

belongs to $\mathrm{L}_{\alpha, \mathbf{q}}^{\mathrm{p}}\left(\mathbb{R}_{\mathbf{q},+}\right)$ and has the form

$$
f^{\varepsilon, \delta}\left(x ; q^{2}\right)=f *_{\alpha, q} G_{q \varepsilon, q \delta}\left(x ; q^{2}\right)+\mu(\{0\}) f(x) \log _{q}\left(\frac{\delta}{\varepsilon}\right) .
$$

where $\mathrm{G}_{\varepsilon, \delta}$ is given by 4.1.).

Proof. Applying q-Fubini's theorem we get

$$
\begin{aligned}
f^{\varepsilon, \delta}(x) & =\int_{\varepsilon}^{\delta} \int_{0}^{\infty} T_{q, x}^{\alpha} f(a y) d_{q} \mu(y) \frac{d_{q} a}{a} \\
& =\int_{0}^{\infty} \int_{\varepsilon}^{\delta} T_{q, a y}^{\alpha} f(x) \frac{d_{q} a}{a} d_{q} \mu(y) \\
& =f(x) \mu(\{0\}) \log _{q}\left(\frac{\delta}{\varepsilon}\right)+\int_{\widetilde{\mathbb{R}}_{q},+} \int_{\varepsilon y}^{\delta y} T_{q, x}^{\alpha} f(a) \frac{d_{q} a}{a} d_{q} \mu(y) \\
& =f(x) \mu(\{0\}) \log _{q}\left(\frac{\delta}{\varepsilon}\right)+\int_{\widetilde{\mathbb{R}}_{q},+} T_{q, x}^{\alpha} f(a)\left(\int_{q \frac{a}{\delta}}^{q \frac{a}{\varepsilon}} \frac{d_{q} a}{a}\right) d_{q} \mu(y) \\
& =f(x) \mu(\{0\}) \log _{q}\left(\frac{\delta}{\varepsilon}\right)+f *_{\alpha, q} G_{q \varepsilon, q \delta}(x) .
\end{aligned}
$$


From this relation, inequality (58) and Lemma 4.1 we deduce that $f^{\varepsilon, \delta}$ belongs to $L_{\alpha, q}^{p}\left(\mathbb{R}_{\mathbf{q},+}\right)$.

Lemma 4.3. Let $\mu \in \mathcal{M}^{\prime}\left(\mathbb{R}_{\mathbf{q},+}\right)$, then for $\mathrm{f} \in \mathrm{L}_{\alpha, \mathbf{q}}^{2}\left(\mathbb{R}_{\mathbf{q},+}\right)$, we have

$$
\mathcal{F}_{\alpha, q}\left(f^{\varepsilon, \delta}\right)\left(\lambda ; q^{2}\right)=\mathcal{F}_{q}(f)\left(\lambda ; q^{2}\right) K_{q \varepsilon, q \delta}\left(\lambda ; q^{2}\right),
$$

where $\mathrm{K}_{\varepsilon, \delta}$, is the function defined in $(67)$.

Proof. This follows from (59), (67) and (80).

Theorem 4.4. Let $\mu \in \mathcal{M}^{\prime}\left(\mathbb{R}_{\mathrm{q},+}\right)$, be such that the q-integral

$$
c_{\mu, \alpha, q}=\int_{0}^{\infty} \mathcal{F}_{\alpha, q}(\mu)(\lambda) \frac{d_{q} \lambda}{\lambda}
$$

be finite. Then for all $\mathbf{f} \in \mathrm{L}_{\alpha, \mathbf{q}}^{2}\left(\mathbb{R}_{\mathbf{q},+}\right)$, we have

$$
\lim _{\substack{\varepsilon \rightarrow 0 \\ \delta \rightarrow \infty}}\left\|f^{\varepsilon, \delta}-c_{\mu, \alpha, q} f\right\|_{2, \alpha, q}=0 .
$$

Proof. By identity (81) and Theorem 2.4 we have

$$
\begin{aligned}
\left\|f^{\varepsilon, \delta}-c_{\mu, \alpha, q} f\right\|_{2, \alpha, q}^{2} & =\left\|\mathcal{F}_{\alpha, q}\left(f^{\varepsilon, \delta}\right)-c_{\mu, \alpha, q} \mathcal{F}_{\alpha, q}(f)\right\|_{2, \alpha, q}^{2} \\
& =\left\|\mathcal{F}_{\alpha, q}(f)\left[K_{\varepsilon, \delta}-c_{\mu, \alpha, q}\right]\right\|_{2, \alpha, q}^{2} .
\end{aligned}
$$

Or $\lim K_{\varepsilon, \delta}(\lambda)=c_{\mu, \alpha, q}$, for all $\lambda>0$ the result follows from the dominate convergence theo$\varepsilon \rightarrow 0$ $\delta \rightarrow \infty$ rem.

Lemma 4.5. Let $\mu \in \mathcal{M}^{\prime}\left(\mathbb{R}_{\mathrm{q},+}\right)$, be such that the q-integral

$$
\int_{0}^{\infty}|\mu([0, y])| \frac{d_{q} y}{y}
$$

be finite. Then the $\mathbf{q}$-integral $\mathbf{c}_{\mu, \alpha, \mathbf{q}}$ is finite and admits the representation

$$
c_{\mu, \alpha, q}=\int_{0}^{\infty} \mu([0, y]) \frac{d_{q} y}{y} .
$$

Proof. From (76) we have

$$
\mathrm{G}_{\varepsilon, \delta}=\frac{\mu\left(\left[\frac{\chi}{\delta}, \frac{x}{\varepsilon}\right]\right)}{\chi^{2 \alpha+2}}=\mathrm{G}_{\varepsilon}-\mathrm{G}_{\delta}
$$


where

$$
G(y)=\frac{\mu([0, y])}{y^{2 \alpha+2}}
$$

and $\mathrm{G}_{\varepsilon}, \mathrm{G}_{\delta}$ the dilated function of $\mathrm{G}$. Since $\mathrm{G} \in \mathrm{L}_{\alpha, \mathrm{q}}^{1}\left(\mathbb{R}_{\mathbf{q},+}\right)$, we deduce from (62) and (78) that

$$
\begin{aligned}
\mathcal{F}_{\alpha, \mathrm{q}} \mathrm{G}_{\varepsilon, \delta}(\lambda) & =\int_{\varepsilon \lambda}^{\delta \lambda} \mathcal{F}_{\alpha, \mathrm{q}} \mu(\mathrm{a}) \frac{\mathrm{d}_{\mathrm{q}} \mathrm{a}}{\mathrm{a}}-\mu(\{0\}) \log _{\mathrm{q}}\left(\frac{\delta}{\varepsilon}\right) \\
& =\mathcal{F}_{\alpha, \mathrm{q}} \mathrm{G}(\varepsilon \lambda)-\mathcal{F}_{\alpha, \mathrm{q}} \mathrm{G}(\delta \lambda),
\end{aligned}
$$

for all $\lambda>0$. Or (84) implies necessarily $\mu(\{0\})=0$. Hence when $\varepsilon=1$ and $\delta \rightarrow \infty$, a combination of (88) and (57) gives

$$
\mathcal{F}_{\alpha, q} G(\lambda)=\int_{\lambda}^{\infty} \mathcal{F}_{\alpha, q} \mu(a) \frac{d_{q} a}{a}, \text { for all } \lambda>0 .
$$

Now the result follows from Formula (84) by using the continuity of $\mathcal{F}_{\alpha, q}(\mu)$.

Theorem 4.6. Let $\mu \in \mathcal{M}^{\prime}\left(\mathbb{R}_{\mathbf{q},+}\right)$ such that

$$
\int_{0}^{\infty}|\mu([0, y])| \frac{d_{q} y}{y}
$$

is finite and $\mathrm{f} \in \mathrm{L}_{\alpha, \mathbf{q}}^{2}\left(\mathbb{R}_{\mathbf{q},+}\right)$. Then

$$
\lim _{\substack{\varepsilon \rightarrow 0 \\ \delta \rightarrow \infty}}\left\|f^{\varepsilon, \delta}-c_{\mu, \alpha, q} f\right\|_{2, \alpha, q}=0 .
$$

Proof. By (800) and (86) we have

$$
f^{\varepsilon, \delta}=f *_{\alpha, q} G_{\varepsilon}-f *_{\alpha, q} G_{\delta}
$$

where G is as in (87). Equation (91) is now a consequence of Proposition 2.5.

\section{Received: July 2014. Accepted: May 2015.}

\section{References}

[1] A. P. Calderón, Intermediate spaces and interpolation, the complex method,Studia Math, 24 (1964), 113-190.

[2] B. Rubin and E. Shamir, Calderón's reproducing formula and singular integral operators on areal line,Integral Equations Operators Theory, 21 (1995), 77-92.

[3] B. Rubin, Fractional Integrals and Potentials ,Logman, Harlow, (1996). 
[4] M.A. Mourou and K. Trim Ã “ Che, Calderón's reproducing formula associated with the Bessel Operator ,J. Math.Anal. Appl., 219 (1998), 97-109.

[5] P.S. PAthak and G. PAndey, Calderón's reproducing formula for Hankel convolution,Inter. J. Math. and Math. Sci., vol 2006 issue 5, Article ID 24217. P.1-7.

[6] J. L. Ansorena and O. Blasco, Characterization of weighted Besov spaces, Math. Nachr, 171 (1995), 5-17.

[7] G. Gasper and M. Rahman, Basic hypergeometric series, 2nd edn. Cambridge University Press, 2004.

[8] H. Q. Bui, M. Paluszynski and M. H. Taibleson , A maximal function charaterization of weighted Besov spaces-Lipschitz and Triebel-Lizorkin spaces, Studia. Math, 119 (1996), 219-246.

[9] A. Nemri and B. Selmi, Calderón type formula in Quantum Calculus, Indagationes Mathematicae, vol 24, issues 3, (2013), 491-504.

[10] A. Nemri and B. Selmi, Some weighted Besov Spaces in Quantum Calculus, submitted.

[11] Nemri A, Distribution of positive type in Quantum Calculus, J. Non Linear. Math. Phys., 4(2006)566583.

[12] A. Fitouni and A. Nemri, Distribution And Convolution Product in Quantum Calculus, Afri. Diaspora J. Math, $7(2008), \mathrm{n} \tilde{\mathrm{A}}^{-} \hat{\mathrm{A}}_{i} \hat{\mathrm{A}}_{\frac{1}{2}}^{1} 1,39-57$.

[13] A. Fitouni, M. Hamza and F. Bouzeffour, The $q-j_{\alpha}$ Bessel function, J. Approx. Theory 115 (2002), 114-116.

[14] M.HadDad, Hankel transform in Quantum Calculus and applications, fractional Calculus and Applied Analysis, Vol.9,issues 4 (2006), 371-384.

[15] T. H. Koornwinder and R. F. Swarttouw, On q-Analogues of the Fourier and Hankel transforms , Trans. Amer Math. Soc. 333, (1992), 445-461.

[16] T. H. Koornwinder, q-Special functions, a tutorial arXiv:math/9403216v1.

[17] A. Fitouhi, L. Dhaouadi and J. El Kamel, Inequalities in q-Fourier analysis , J. Inequal. Pure Appl. Math, 171 (2006), 1-14.

[18] A. Fitouni and L. Dhaouadi, Positivity of the Generalized Translation Associated to the q-Hankel Transform, Constr. Approx, 34 (2011), 453-472.

[19] V.G. Kac And P. Cheeung, Quantum calculus, Universitext, Springer-Verlag, New York, (2002). 